Skeletal muscle vibrates laterally during voluntary and evoked muscle contractions. We hypothesized that the vibration amplitude from evoked muscle twitches is directly related to evoked twitch force from fatiguing muscle. To test the hypothesis, vibrations produced by evoked muscle twitches were recorded during short (5-second) rest periods as the muscle was intermittently exercised with voluntary contractions. Trials were performed at $30 \%, 50 \%$, and $70 \%$ of maximal voluntary contraction. Evoked muscle twitches eliminated the problems of motivation and tremor that complicate sound and vibration measurements during voluntary contractions. Results from the first dorsal interosseus hand muscle in 11 normal adult volunteers revealed that the vibration amplitude is highly correlated $\left(r^{2}=0.93\right.$, at $70 \%$ MVC, $r^{2}=0.97$, at $50 \%$ MVC; $r^{2}=0.85$, at $30 \%$ MVC) with force. Both potentiation and reduction of force with exercise were accompanied by parallel changes in vibration amplitude, as measured with an accelerometer. Compound muscle action potentials did not increase with exercise-induced twitch potentiation, and did not correlate as highly with force during fatigue. Key words: muscle fatigue $\bullet$ muscle sounds $\bullet$ acoustic myography

MUSCLE \& NERVE 15:303-309 1992

\title{
MUSCLE FATIGUE MEASURED WITH EVOKED MUSCLE VIBRATIONS
}

\author{
DANIEL T. BARRY, MD, PhD, TIMOTHY HILL, MD, and DUKJIN IM, MD
}

Contracting skeletal muscle vibrates and emits sounds that are related to the force of contraction. ${ }^{6,19}$ The vibrations are an intrinsic component of the contraction process. ${ }^{1,13}$ Frog muscle studies in vitro have shown that the vibrations occur at the mechanical resonant frequency of the muscle. $^{4,5}$ The resonant frequency increases during a twitch, because both tension and muscle stiffness increase as more cross-bridges attach. Joint timefrequency transformations have been used to track the frequency change as a function of time during a contraction. ${ }^{5}$

Note added in proof: At a special session for muscle sounds during the IEEE BMES annual meeting in Orlando, Fiorida December 3, 1990, the group present agreed to call the study of muscle sounds "vibromyography" or VMG rather than acoustic myography, acceleromyography, muscle sounds, or other various terms currently in use.

From the Department of Physical Medicine and Rehabilitation, University of Michigan Medical Center, Ann Arbor, Michigan

Acknowledgments: This work was supported by grant NS-01017 from the National Institutes of Health. Parts of this work were presented at the 1990 American Academy of Physical Medicine and Rehabilitation Meeting in Phoenix, Arizona.

Address reprint requests to Daniel T. Barry, MD, PhD, Department of Physical Medicine and Rehabilitation, University of Michigan Medical Center, Box 0042, Ann Arbor, MI 48109-0042

Accepted for publication March 13, 1991.

CCC 0148-639X/92/030303-07 $\$ 04.00$

(c) 1992 John Wiley \& Sons, Inc.
Human muscle twitches also produce vibrations of the muscle. The vibrations propagate to the skin surface and cause the skin to vibrate. The skin vibrations cause longitudinal pressure waves (sounds) to propagate through the air. Some frequency components of the sounds are audible to human ears, and the full range of frequencies can be recorded with a wideband microphone.

Previous work with microphones revealed that human muscle vibrations, produced during evoked muscle twitches, have characteristic waveforms with measurable latencies and amplitudes. ${ }^{3,12,15}$ Using evoked acoustic signals eliminates the problems of motivation and microphone stability that are encountered in voluntary acoustic signals. Furthermore, evoked acoustic signals can be generated, displayed, and analyzed in a manner similar to electrical nerve conduction studies.

Skeletal muscle sounds produced during contraction have been used to measure force, monitor fatigue, control a prosthesis, and in the diagnosis of muscle disease. ${ }^{6-8,13,14,20,21}$ Problems encountered in recording voluntary muscle sounds include tremor artifact and measurement of subject motivation. Tremor and motivation problems are eliminated by using supramaximal percutaneous nerve stimulation and recording muscle sounds during a twitch. ${ }^{3,12,15}$ Another problem with most previous studies was that the transducers were calibrated in electrical units rather than physiologic 
units (e.g., acceleration, velocity, pressure, or displacement).

In the present study, an accelerometer was used instead of a microphone so the signal was calibrated in physiologic units (meters per second per second) rather than transducer-dependent units. Previous work has been done with accelerometers in measuring muscle vibrations. ${ }^{16,17}$ However, the data are generally reported in normalized units rather than physiologic units.

Simultaneously recording muscle sound and force during a maximal voluntary isometric contraction reveals that the sound amplitude declines with force as fatigue occurs. ${ }^{6,14,18}$ The ratio of acoustic signal amplitude to EMG signal amplitude declines with fatigue and may be useful as a measure of the "efficiency" of electrical excitation.

As a subject becomes fatigued, physiological tremor can interfere with the acoustic signal. Tremor artifact can be effectively eliminated by a high-pass filter, but some of the sound signal will be lost because the frequency bands overlap. ${ }^{14}$ One possible alternative is to use two sound transducers, one over the muscle of interest and the other over a relaxed muscle in the same extremity, and differentially amplify the two signals. Another alternative is to allow the subject to relax periodically during the protocol, and to supramaximally stimulate the peripheral nerve to the muscle during relaxation. The evoked twitch has no tremor artifact and has the additional advantage of eliminating motivational factors in the measurement.

\section{METHODS}

Eleven adult volunteers without known neuromuscular disease were recruited for the study, and provided informed consent in accordance with a protocol approved by the University of Michigan Human Subjects Committee. Each subject was seated comfortably with their right hand in a modified wrist-hand orthosis that held the second digit extended and secured the hand and wrist. Hand temperatures were maintained between $32^{\circ} \mathrm{C}$ and $34^{\circ} \mathrm{C}$, measured with a thermistor on the dorsal surface of the hand, by using silicone hot packs when necessary.

Muscle twitches were produced by supramaximal percutaneous ulnar nerve stimulation. Compound muscle action potentials (CMAPs) were recorded with an active electrode over the belly of the first dorsal interosseus hand (FDIH) muscle and the reference electrode over the interphalangeal joint of the thumb. To minimize size and mass, the active CMAP electrode was made of a tiny piece of metal foil with sticky conductive gel.

The CMAP was amplified with a TECA TD-20 electromyograph, with filter settings of $2 \mathrm{~Hz}$ to 10 $\mathrm{KHz}$. Lateral acceleration of the muscle belly was recorded with an Entran model EGA-125-50D accelerometer (Entran, Fairfield, NJ, sensitivity 0.41 $\mathrm{mV} /$ (meter per second per second) at $15-\mathrm{V}$ excitation, mass of $0.5 \mathrm{~g}$ ) attached with thin doublesided tape adjacent to the active CMAP electrode. The accelerometer was amplified by a Grass P16 amplifier (DC to $2 \mathrm{kHz}$ bandpass, Grass Instrument Co., Quincy, MA). A half-inch loop of cloth held the second digit in extension and provided restraint so that the muscle twitches were approximately isometric for the FDIH. Force was recorded with a load cell (Western Load Cell Co., Encinio, CA, model \#65) attached to the cloth loop. The force was amplified with a Grass P16 amplifier (DC to $2 \mathrm{kHz}$ bandpass) coupled to a $\mathrm{DC}$ fixed gain amplifier, and an antialiasing $500-\mathrm{Hz}$ Butterworth low-pass filter.

The force measured was the net abduction torque of the second digit. The torque is the resultant of ulnar innervated muscle contractions and the moment arm from the muscle insertions to the cloth loop, not just the force generated by the FDIH. We assumed that the signal was dominated by the FDIH component. This assumption was supported by the smooth rise and decay of torque, similar to the waveform expected for force from a single muscle twitch.

The maximal voluntary contraction (MVC) level was determined at the beginning of each testing session by asking the subject to exert abduction force with the second digit. Force was maintained for about 2 seconds. The procedure was done twice, and the highest value was used. Visual feedback of the force exerted was provided on an oscilloscope screen. After MVC was determined, the supramaximal stimulation level was determined by increasing the stimulation to $20 \%$ above the intensity to where the CMAP and force no longer increased. After a 2-minute rest, 2 twitches were evoked with stimulations. These two stimulations were averaged to determine the baseline twitch amplitude. Subjects then voluntarily exerted force at a target level equal to $30 \%, 50 \%$, or $70 \%$ of $\mathrm{MVC}$ - the level depended on the particular trial being performed.

Visual feedback of force on the oscilloscope screen helped the subject to maintain a steady force at the target level. When the subject was unable to hold the target force, they exerted the maximum force possible. This was enhanced by 
visual feedback of the root mean squared value of the surface electromyography signal on a voltmeter. After 25 seconds of activity, subjects rested for 5 seconds, and they resumed the target level of force. At 4 seconds into each 5 -second rest period, a single twitch was evoked by stimulation. Once the subject was unable to maintain the target force, the subject was instructed to provide the maximum force able to generate for the 25second activity period. When the subject was no longer able to maintain the target force for at least the first 5 seconds of activity, then 3 more activity periods were performed and the trial ended. No subject performed more than one trial in any one day.

Each channel of data (CMAP, force, accelerometer) was digitized with sampling rates of either 6.250 or $8.333 \mathrm{kHz}$ and 12-bit resolution. No data waveforms contained frequency components greater than $3.0 \mathrm{kHz}$, so the sampling rates were adequate. The data were subsequently analyzed digitally using a Zenith 386 PC (Zenith Data Systems, Chicago, IL).

\section{RESULTS}

The acceleration signal from a typical muscle twitch was an oscillating waveform containing sev-

A

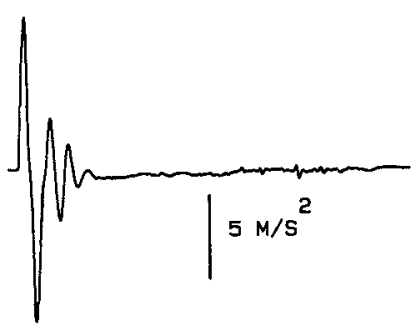

$50 \mathrm{~ms}$

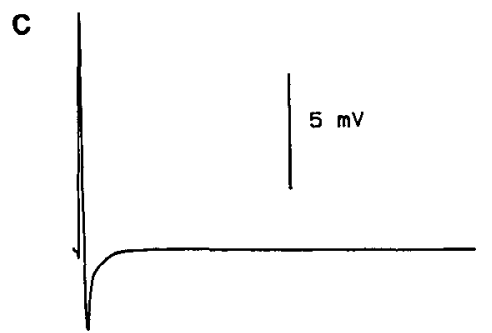

eral cycles that decayed in amplitude with time (Fig. 1). The onset of the acceleration signal was after the onset of the compound muscle action potential (CMAP) and before the onset of force production. The acceleration waveform shape and timing closely resemble the signal seen with hydrophone recordings of frog muscle sounds in vitro. ${ }^{1,13}$

Figure 2 shows an example of acceleration, force, and CMAP for a single subject at 4 stages of a single trial. All 3 signals declined in amplitude with fatigue. In the acceleration signal, the first oscillation cycle amplitude declined more than the amplitude of later half-cycles as fatigue progressed. In other words, fatigue caused the energy of acceleration to shift to later times in the waveform. The acceleration waveforms produced with a subject's hand unrestrained by an orthosis tend to peak later in the waveform (unpublished data), resembling a fatigued isometric twitch acceleration waveform more than an unfatigued one. The CMAP became broadened and sometimes doublehumped with fatigue.

Twitch force consistently potentiated after the first 25-second exercise period, compared with the baseline twitch force (Fig. 3). The increase is accompanied by an increase in the amplitude of the acceleration signal, but the CMAP did not in-

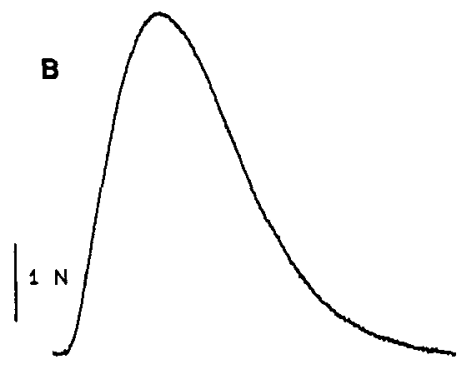

FIGURE 1. Waveforms are shown from a typical unfatigued isometric contraction of the first dorsal interosseus hand muscle. All 3 waveforms are on the same time scale. (A) Acceleration waveform $\left(\mathrm{m} / \mathrm{s}^{2}=\right.$ meters per second per second). (B) Force $(\mathrm{N}=$ Newtons). (C) Compound muscle action potential. 
A

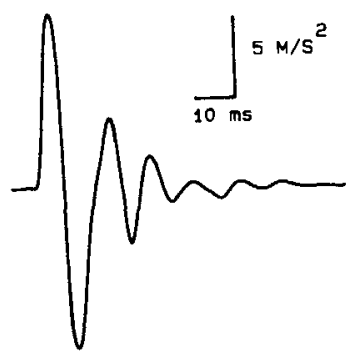

$\mathbf{B}$

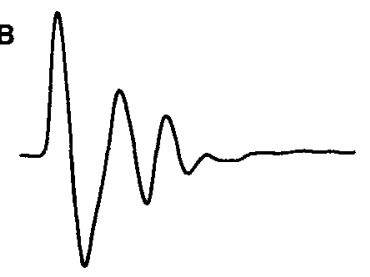

C
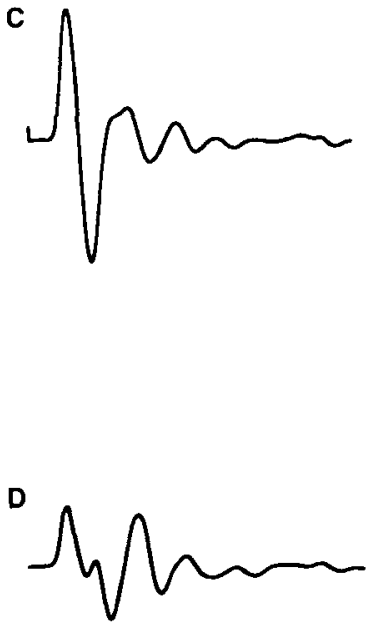
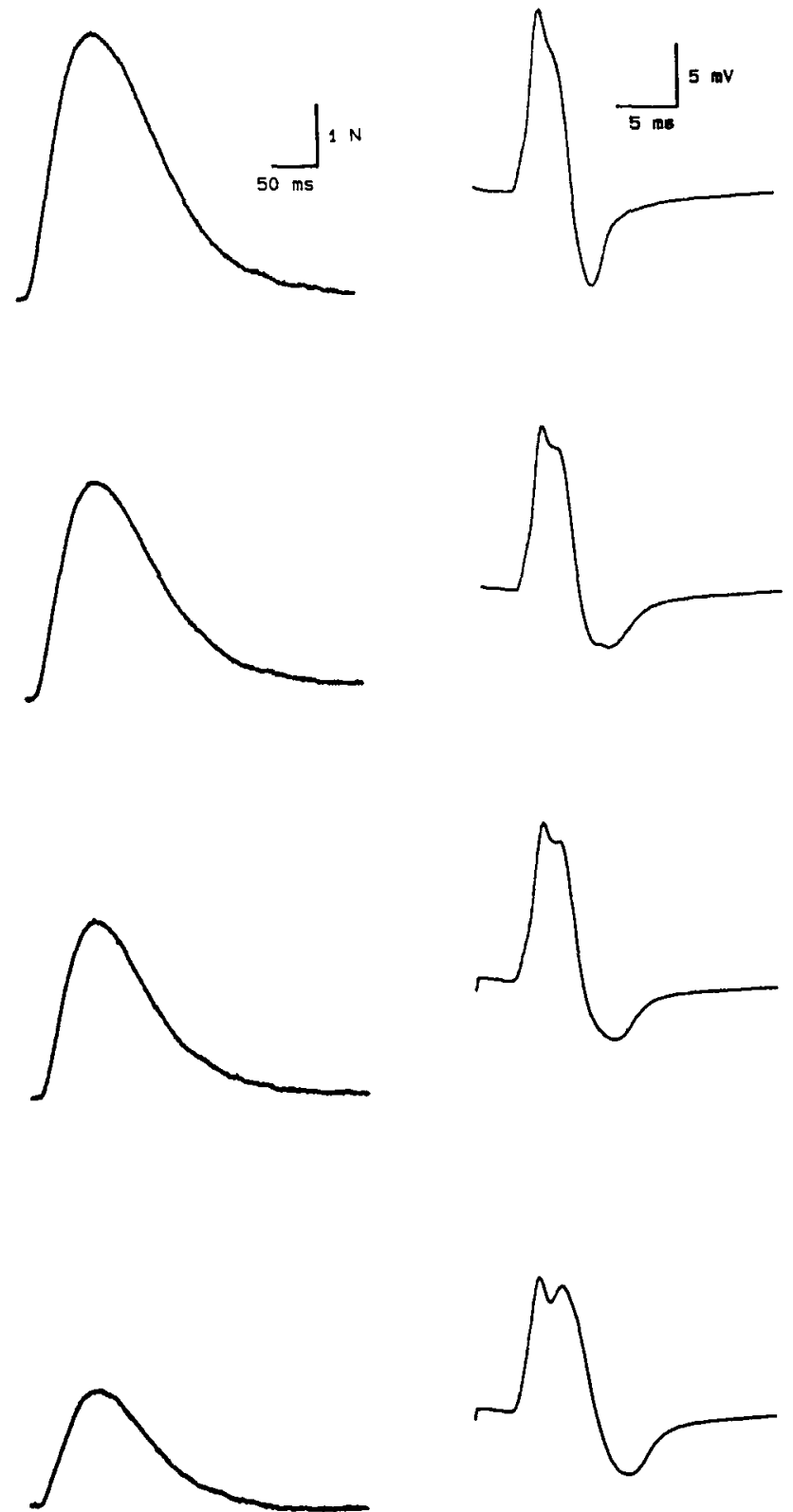

FIGURE 2. A series of waveforms is shown during a fatigue trial. These data are from a different subject than the data in Figure 1. Note that the signals are not on the same time scale. (A) Acceleration, force, and compound muscle potential after the first 25-second exercise period. (B) Same signals with twitch force reduced by $20 \%$. (C) Same signals with twitch force reduced by $30 \%$. (D) Same signals with twitch force reduced by $55 \%$.

crease. Subsequent exercise periods produced fatigue that was revealed by the decline in twitch force (Fig. 3).

For all trials, the acceleration signal was more highly correlated with twitch force than the CMAP (Table 1), and the overall decline in CMAP amplitude was less than the overall decline of force or acceleration. In general, the CMAP am- plitude did not decline as fast as force or acceleration in the initial stages of fatigue. These relationships are revealed by least mean squared linear regression of CMAP amplitude to force and of acceleration amplitude to force (Table 1). In all 3 trials, the slope of the CMAP-to-force relationship was less than half the slope of the acceleration-toforce relationship. 
Table 1. Regression coefficients.

\begin{tabular}{llll}
\hline Trial & $r^{2}$ & $a$ & $b$ \\
\hline 70\% MVC & & & \\
Acceleration & 0.92 & 1.1 & 0.0 \\
CMAP & 0.24 & 0.3 & 0.7 \\
50\% MVC & & & \\
Acceleration & 0.97 & 0.9 & 0.1 \\
CMAP & 0.88 & 0.4 & 0.6 \\
30\% MVC & & & \\
Acceleration & 0.85 & 0.7 & 0.3 \\
CMAP & 0.76 & 0.3 & 0.6 \\
\hline
\end{tabular}

Results of least mean squared linear regression for acceleration and CMAP results as functions of force (see Fig. 4); $r$ is the correlation coefficient. $\mathrm{a}$ and $\mathrm{b}$ are the coefficients of the linear equation $\mathrm{ax}+\mathrm{b}$. relating acceleration or CMAP amplitude to force. Analysis of variance showed that linear regression is appropriate $(P<0.01)$ for all fits except the CMAP plot for the $70 \%$ MVC trial $(\mathrm{P}=0.15)$.

\section{DISCUSSION}

The oscillating appearance of the acceleration signal is consistent with in vitro experiments on frog muscle, where the muscle sound signal recorded with hydrophones is proportional to the lateral acceleration of the muscle. ${ }^{1,43}$ Human muscle sound waveforms recorded in vivo with air- coupled microphones appear biphasic, without the oscillations seen here. Air-coupled microphones do not reveal the oscillations because the pressure produced in the air chamber is proportional to skin displacement rather than acceleration. Additional distortions can occur due to cutoff frequencies of the transducer and/or mechanical binding of the skin by a massive transducer or excessive application pressure. Previous work has shown that integrating the acceleration signal twice (to obtain displacement) does produce a signal that is similar to the air-coupled microphone waveform. ${ }^{2}$

The increase in twitch force after the first exercise period is due to posttetanic potentiation. The increase in the acceleration signal together with force is consistent with the hypothesis that the acceleration signal reflects the mechanical activity of the muscle. The CMAP does not increase, because all of the muscle fibers were contributing to the baseline CMAP and there is no increase in size or number of muscle fiber action potentials with muscular posttetanic potentiation.

The cause of the shift of high-amplitude oscil-
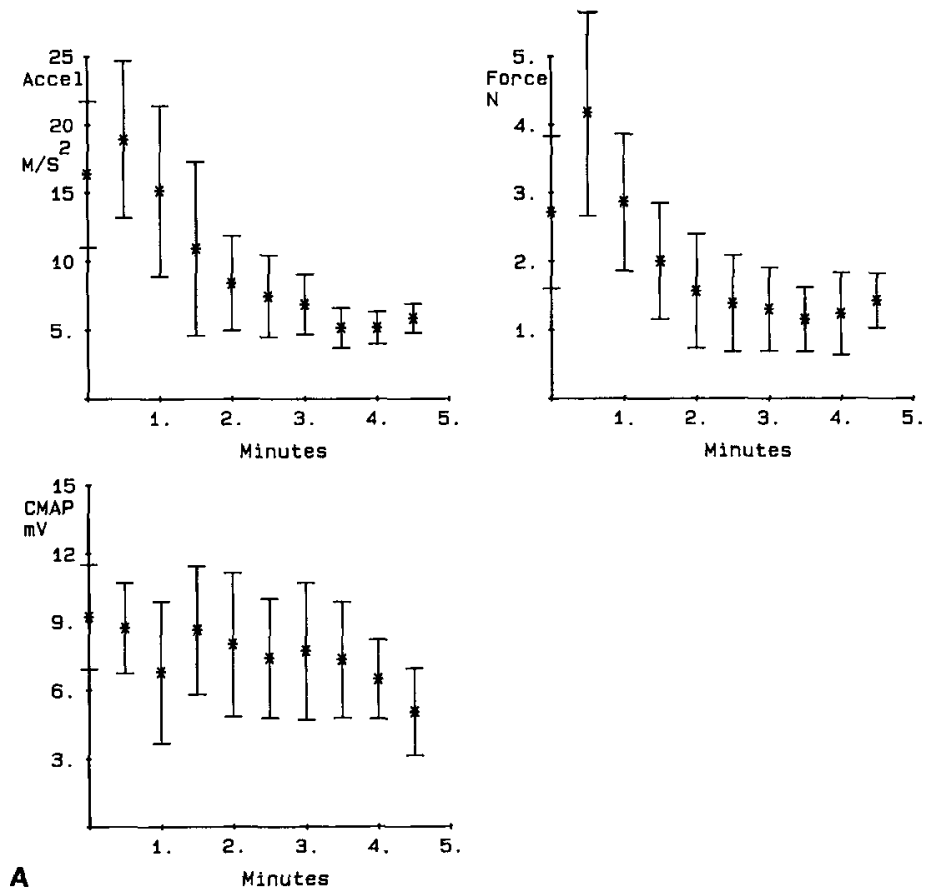

FIGURE 3. Acceleration, force, and CMAP are shown as functions of time during the fatigue trials. Data points occur every 30 seconds, corresponding to the stimulation times during the trials. The first point in each record is from the stimulation prior to exercise. Note the increase in force and acceleration amplitude due to potentiation after the first exercise period of each trial (second data point of each graph). The data are averaged values for all subjects. Error bars denote standard deviation. (A) $70 \%$ maximum voluntary contraction (MVC) trials. 

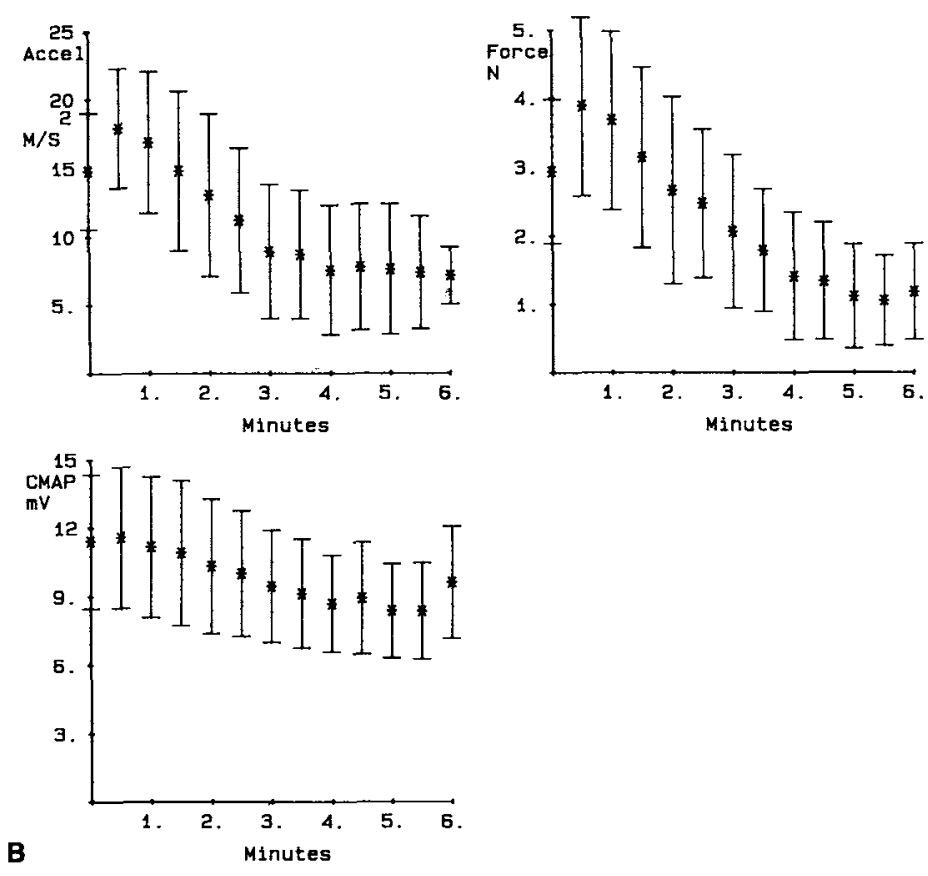

FIGURE 3. (B) $50 \%$ MCV trials.
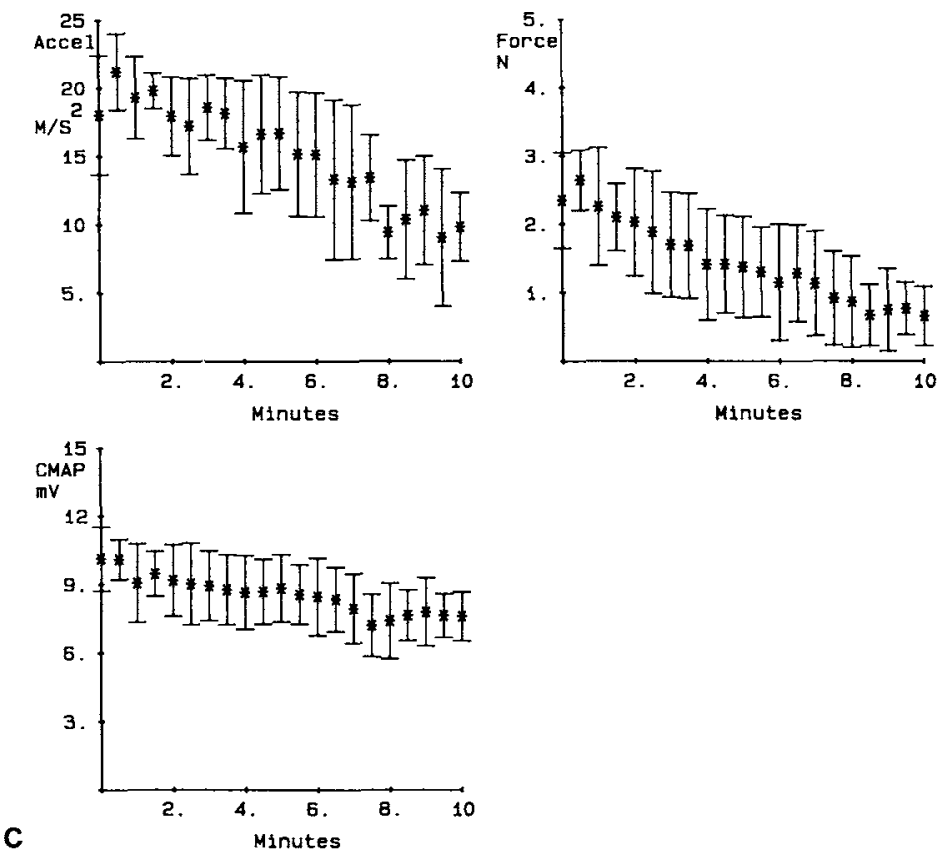

FIGURE 3. (C) $30 \%$ MCV trials.

lations from the beginning of the acceleration signal to later in the waveform is unknown. One possibility that cannot be ruled out with these experiments is that another ulnar-innervated muscle contributes to the signal to a proportionately greater extent as the FDIH fatigues. This may also explain the double hump that sometimes appeared in the CMAP after fatigue (Fig 3). However, the shift in high amplitude from early to late in the acceleration waveform has also been seen with some isolated frog muscle preparations after fatigue induced with stimulations. 
There may be multiple vibratory modes that are selectively enhanced or suppressed as the muscle fatigues. Alternatively, the change in contractile speeds produced by fatigue may cause lateral acceleration to peak later in the waveform. The explanation for this effect will require further investigation.

The decline in CMAP amplitude may be due to increased dispersion between muscle fiber action potentials in different muscle fibers as conduction velocity declines with fatigue. However, some failure of neuromuscular junctions or terminal nerve ends cannot be ruled out on the basis of these experiments. Surface EMG recordings during voluntary fatiguing contractions depend on the level and duration of contraction, but at moderate levels of contraction the EMG amplitude typically does not decline, suggesting that neuromuscular junction failure does not usually accompany fatigue during voluntary contractions.9,10,11
In any case, the CMAP is a reasonable measure of the electrical activation of muscle fibers. If the acceleration signal accurately represents the mechanical response of muscle fibers, then the ratio of acceleration amplitude to CMAP amplitude should provide a measure of excitation-contraction coupling without the need for a force transducer apparatus.

In summary, the evoked signal eliminates artifacts of subject motivation and tremor that complicate vibration measurements during voluntary contractions. The amplitude of lateral acceleration of the FDIH muscle belly was highly correlated with supramaximal evoked twitch force during fatigue, reflecting potentiation as well as reduction of twitch force with exercise. The acceleration waveform changed during exercise in a characteristic way that may reflect fatigue-induced changes in contractile properties of the muscle.

\section{REFERENCES}

1. Barry DT: Acoustic signals from frog skeletal muscle. Biophys J 1987:51:769-773.

2. Barry DT: Evaluation of transducers for recording muscle sounds. Muscle Nerve 1990;13:872 (abstr).

3. Barry DT: Muscle sounds from evoked muscle twitches. Arch Phys Med Rehab 1991;72(8):573-575.

4. Barry DT, Cole NM: Fluid mechanics of muscle vibrations. Biophys J 1988;53:899-905.

5. Barry DT, Cole NM: Mechanical vibrations occur at the resonant frequency of skeletal muscle. IEEE Biomed Eng. $1990 ; 37: 525-531$.

6. Barry DT, Geiringer SR, Ball RD: Acoustic myography: a non-invasive monitor of motor unit fatigue. Muscle Nerve 1985;8:189- 194

7. Barry DT, Gordon KE, Hinton GG: Acoustic and surface EMG diagnosis of pediatric muscle disease., Muscle Nerve $1990 ; 13: 286-290$.

8. Barry DT, Lconard JA, Gitter AJ, Ball RD: Acoustic myography as a control signal for an externally powered prosthesis. Arch Phys Med Rehabil 1986;67:267-269.

9. Bigland-Ritchie B: EMG and fatigue of human voluntary and stimulated contractions in CIBA Symposium 82, I.ondon, Pitman Medical, 1981, pp 130-156.

10. Bigland-Ritchie B, Furbush F, Woods JJ: Fatigue of intermittent, submaximal voluntary contractions: Central and peripheral factors in different muscles. J Appl Physiol 1986;61:421-429.

11. Bigland-Ritchie $B$, Woods $\mathrm{J}$ : Changes in muscle contractile properties and neural control during human muscle fatigue. Muscle Nerve 1984;7:691-699.

12. Bolton CF: Recording sound from human skeletal muscle: technical and physiological aspects. Muscle Nerve 1989;12: $126-134$.
13. Frangioni JV, Kwan-Gett TS, Dobrunz LE, McMahon TA: The mechanism of low-frequency sound production in muscle. Biophys $J$ 1987;51:775-783.

14. Goldenberg MS, Yack HJ, Ceray FJ, Button HW: Acoustic myography as an indicator of force during sustained contractions of a small hand muscle. $J$ Appl Physiol 1991; $70: 87-91$.

15. Hufschmidt A: Acoustic phenomena in the latent period of skeletal muscle: a simple method for in-vivo measurement of teh electro-mechanic latency (EML). Pflugers Arch $1985 ; 404: 162-165$.

16. Keidel M, Keidel WD: The computer-vibromyography as a biometric progress in studying muscle function. Biomedizinische Technik 1989;34:107-116.

17. Lammert $O$, Jorgensen $F$, Einer-Jensen NE Accelerometermyography (AMG) I: Method for measuring vibrations from isometrically contracted muscles, in Komi PV (cd): $B i$ omechanics $V$-A. Baltimore, MD, University Park Press, 1976 , pp $150-156$.

18. Orizio C, Perini R, Veicsteinas A: Changes of muscular sound during sustained isometric contraction up to exhaustion. J Appl Physiol 1989;66:1593-1598.

19. Oster $(x$, Jaffee JS: Low frequency sounds from sustained contraction of human skeletal muscle. Biophys $J$ 1980;30: $119-127$.

20. Stokes IAF, Moffroid MS, Rush S, Haugh LD: Comparison of acoustic and electrical signals from erectores spinae muscles. Muscle Nerve 1988;11:331-336.

21. Wee AS, Ashley RA: Vibrations and sounds produced during sustained voluntary muscle contraction. Electromyogr Chin Neurophysiol 1989;29:333-337. 